\title{
Functional connectivity of the anterior insula associated with intolerance of uncertainty in youth
}

\author{
Mariah DeSerisy $^{1}$ (D) - Alec Musial ${ }^{1} \cdot$ Jonathan S. Comer ${ }^{2} \cdot$ Amy K. Roy $^{1,3}$ \\ Published online: 2 March 2020 \\ (C) The Psychonomic Society, Inc. 2020
}

\begin{abstract}
Intolerance of uncertainty (IU) is a trait characteristic marked by distress in the face of insufficient information. Elevated IU has been implicated in the development and maintenance of anxiety disorders, particularly during adolescence, which is characterized by dramatic neural maturation and the onset of anxiety disorders. Previous task-based work implicates the bilateral anterior insula in IU. However, the association between anterior insula intrinsic functional connectivity (iFC) and IU has not been examined in adolescents. Fifty-eight healthy youth (mean age $=12.56 ; 55 \%$ boys) completed the Intolerance of Uncertainty Scale for Children (IUSC-12) and a 6-minute resting state fMRI scan. Group-level analyses were conducted using a randomeffects, ordinary least-squares model, including IUSC-12 scores (Total, Inhibitory subscale, Prospective subscale), and three nuisance covariates (age, sex, and mean framewise displacement). IUSC-12 Inhibitory subscale scores were predictive of iFC between the left and right anterior insula and right prefrontal regions. IUSC-12 Prospective subscale scores significantly predicted iFC between the anterior insula and the anterior cingulate cortex. IUSC-12 total scores did not predict significant iFC of the bilateral anterior insula. Follow-up analyses, including anxiety (MASC Total Score) in the models, failed to find significant results. This could suggest that the associations found between IUSC-12 scores and anterior insula iFC are not unique to IU and, rather, reflect a broader anxiety-related connectivity pattern. Further studies with larger samples are needed to tease apart unique associations. These findings bear significance in contributing to the literature evaluating the neural correlates of risk factors for anxiety in youth.
\end{abstract}

Keywords Intolerance of uncertainty $\cdot$ Anterior insula $\cdot$ Anxiety $\cdot$ fMRI $\cdot$ Adolescence $\cdot$ Uncertainty

Intolerance of uncertainty (IU) is a transdiagnostic vulnerability factor that has been defined as "an individual's dispositional incapacity to endure the aversive response triggered by the perceived absence of salient, key, or sufficient information, and sustained by the associated perception of uncertainty" (Carleton, 2016; p. 31). In healthy adults, uncertainty, or the feeling of "not knowing," has been shown to intensify both

Electronic supplementary material The online version of this article (https://doi.org/10.3758/s13415-020-00780-x) contains supplementary material, which is available to authorized users.

Mariah DeSerisy

mdeserisy@fordham.edu

1 Fordham University, Dealy Hall 436, 441 East Fordham Road, Bronx, NY 10458, USA

2 Florida International University, Miami, FL, USA

3 Hassenfeld Children's Hospital of New York at NYU Langone Child Study Center, New York, NY, USA positive and negative emotional experiences (Bar-Anan, Wilson, \& Gilbert, 2009). Intolerance of this feeling of uncertainty is said to underlie negative affectivity, negative problem orientation, and reduced resiliency, while increasing the probability that an individual will engage in maladaptive coping strategies, such as worry or alcohol use, to address negative affect (Bar-Anan et al., 2009; Einstein, 2014; Freeston, Rhéaume, Letarte, Dugas, \& Ladouceur, 1994). As such, IU is an important risk factor in the development and maintenance of a range of psychiatric disorders. For example, in adult clinical populations, IU has been shown to influence causally the development and maintenance of anxiety disorders (Dugas \& Ladouceur, 2000) and to contribute to transdiagnostic anxiety across mental health disorders, including eating disorders (Kesby, Maguire, Brownlow, \& Grisham, 2017), depression (Mahoney \& McEvoy, 2012), substance use disorders (Banducci, Bujarski, Bonn-Miller, Patel, \& Connolly, 2016), obsessive compulsive disorder (Tolin, Abramowitz, Brigidi, \& Foa, 2003), and psychosis (White \& Gumley, 2010). 
As in adults, elevated IU has been observed in children and adolescents across a range of disorders, including anxiety and attention-deficit/hyperactivity disorder (ADHD; Gramszlo, Fogleman, Rosen, \& Woodruff-Borden, 2018). Additionally, the relationships between IU and pathological factors, such as anxiety and maladaptive coping, appear to peak in adolescence as children develop the cognitive capacity to anticipate, understand, and elaborate on potential negative outcomes (Barahmand, 2008; Dugas, Laugesen, \& Bukowski, 2012; Grist \& Field, 2012; Vasey \& Crnic, 1994). Given that the transition from childhood to adolescence also involves significant neural changes, investigation of brain mechanisms underlying general risk factors, such as IU, can inform etiological models of the emergence of psychopathology during this critical developmental period.

Individual differences in IU have been shown to be associated with alterations in task-based activation of several brain regions, including the amygdala, prefrontal cortex, dorsal anterior cingulate (dACC), and anterior insula (AI) (Gorka, Nelson, Phan, \& Shankman, 2016; Schienle, Kochel, Ebner, Reishofer, \& Schafer, 2010; Simmons, Matthews, Paulus, \& Stein, 2008). The most consistent of these findings has been observed in the AI, which plays a specific role in individuals' self-reflective awareness of their own emotional experiences (Seeley et al., 2007; Wever, Smeets, \& Sternheim, 2015), particularly during highly salient, emotionally arousing situations, such as that experienced by individuals with elevated IU during periods of uncertainty (Eckert et al., 2009; Phan, Wager, Taylor, \& Liberzon, 2002). The AI, along with other regions, including the AACC, is a part of the brain's salience network, which supports decision making, ambiguity, and novelty (Uddin, 2017), processes shown to be disrupted among healthy adults with high IU (Carleton et al., 2016; Flores, Lopez, Vervliet, \& Cobos, 2018; Hunt, Cooper, Hartnell, \& Lissek, 2019; Luhmann, Ishida, \& Hajcak, 2011). Task-based functional imaging studies in healthy adults have demonstrated that hyperactivation in the $\mathrm{AI}$ in response to unpredictable or ambiguous stimuli is associated with greater uncertainty and IU (Gorka et al., 2016; Shankman et al., 2014; Simmons et al., 2008). A task-based functional connectivity study of healthy adults found that individuals who prefer to avoid making decisions in the face of uncertainty, which may suggest IU, show greater dACC-insula coupling during a decision-making task than those who do not avoid (Jung et al., 2014). This supports involvement of the broader salience network in IU, which also is observed in task-based studies of healthy and anxious adolescents showing greater IU associated with hyperactivation of the AACC in response to uncertainty (Krain et al., 2006, 2008). To date, no resting-state studies have been performed to examine the associations between IU and the intrinsic functional connectivity (iFC) of the $\mathrm{AI}$ and related salience network in adults or pediatric samples. Given that IU may serve as a risk factor for psychopathology, and that disruption in the salience network and its connectivity with other canonical networks have been theorized to contribute to the development and maintenance of adult psychopathology (Abrams et al., 2019; Goodkind et al., 2015; Han et al., 2019; Manoliu et al., 2013; Menon, 2011; Peters, Dunlop, \& Downar, 2016; White, Engen, Sørensen, Overgaard, \& Shergill, 2014; Yang et al., 2019), this seems like an important next step.

To inform etiologic models of psychopathology and contribute to the growing literature evaluating transdiagnostic risk factors, such as IU, and their neural correlates, the current study addresses gaps in the literature by assessing the association between IU and the iFC of the AI in a sample of healthy children and adolescents. As described above, adolescence is a particularly salient developmental period in which to study IU in the brain, because it is associated with significant normative social, behavioral, and neural maturation (Steinberg \& Morris, 2001), as well as increases in the psychopathology onset (Kessler et al., 2005; Merikangas et al., 2011). Healthy youth were selected to assess more clearly the associations between IU and $\mathrm{AI}$ iFC without the confounding effects of psychopathology and thus have the potential to inform predictive models of risk and vulnerability. Adult findings suggest that increased connectivity between the AI and the dACC may be associated with increased IU (Jung et al., 2014). Furthermore, task-based work suggests increased coactivation of the AI and the AACC among individuals with elevated IU (Gorka et al., 2016). Based on these findings, we predict that elevated IU in youth will be associated with increased AIdACC connectivity. However, given that this hypothesis is based on adult task-based work, and that this study is the first to explore these findings in children and adolescents using resting-state methods, a whole-brain approach was used to evaluate associations between IU and AI functional connectivity with the entire brain.

Recent bifactor models in children and adolescents suggest that the broader construct of IU, as assessed by the Intolerance of Uncertainty Scale (IUS) and the Intolerance of Uncertainty Scale for Children (IUSC; Comer et al., 2009; Cornacchio et al., 2018; Freeston et al., 1994), may be better explained by a general factor along with two subfactors: "Inhibitory IU" and "Prospective IU" (Carleton, Norton, \& Asmundson, 2007; Cornacchio et al., 2018). Prospective IU reflects an inherently future orientation and refers to apprehensive anxiety or the desire for predictability prior to feared events. Inhibitory IU, in contrast, is more present focused and refers to the inhibition of behaviors due to uncertainty or uncertainty paralysis. This model structure has been supported by confirmatory factor analyses in adolescent samples (Boelen, Vrinssen, \& van Tulder, 2010; Dekkers, Jansen, Salemink, \& Huizenga, 2017) and has been clinically validated such that each factor is associated with unique psychopathological symptoms (Boelen et al., 2010; Cornacchio et al., 2018). For 
example, Prospective IU has been associated with harm avoidance and worry, whereas Inhibitory IU has been more associated with phobic responses and feared based processes (Cornacchio et al., 2018). Of note, no studies have evaluated the subfactors of IU in the adolescent brain. In light of this, we completed exploratory analyses evaluating AI iFC associations with the general IU factor as well as with the two IU subfactors of Prospective and Inhibitory IU.

\section{Methods and materials}

\section{Participants}

Sixty-two healthy youth ages 8-17 years were recruited from the community to participate in resting state functional MRI research studies at a major medical center. Youth were included in the current study if they (1) failed to meet criteria for any current psychiatric disorders on the basis of a semistructured interview (ADIS-IV-C (Silverman \& Albano, 1996) or KSADS-PL (Kaufman et al., 1997), (2) completed the IUSC, and (3) completed an MRI scan session with at least one structural and one complete resting state scan. Children with IQ scores of less than 75 or MRI contraindications, including history of concussion, metal in the body, etc., were excluded. Four children were excluded due to incomplete or unusable scan data (mean framewise displacement (FD) $\geq$ $0.3 \mathrm{~mm}$ or $\max \mathrm{FD} \geq 1 \mathrm{~mm}$ ). The final sample included 58 healthy children and adolescents (32 boys, $41 \%$ non-Hispanic Caucasian, 14\% Hispanic), ages $8-17$ years $(M=12.56$; $S D=$ 2.86; Table 1).

Parental consent and child assent were obtained. The parent studies from which this final sample was obtained were approved by the New York University (NYU) School of Medicine Institutional Review Board and NYU Committee on Activities Involving Human Subjects.

\section{Measures}

Children and adolescents completed the Intolerance of Uncertainty Scale for Children (IUSC; Comer et al., 2009). In line with recent recommendations based on the observed factor structure of IU in youth (Cornacchio et al., 2018), a shortened (12-item, minimum score $=12$, maximum score $=$ $60)$ version of the scale was used. The IUSC-12 total score, as well as the IUSC-12 Prospective ( 7 items, minimum score $=7$, maximum score $=35$ ) and the IUSC-12 Inhibitory ( 5 items, minimum score $=5$, maximum score $=25$ ) subscales were included. In the current sample, internal consistency was strong for the IUSC-12 total score $(\alpha=0.80)$ and the IUSC12 Prospective subscale $(\alpha=0.76)$, and somewhat lower for the IUSC-12 Inhibitory subscale $(\alpha=0.64)$. A subset of participants $(\mathrm{N}=55)$ also completed the Multidimensional
Table 1. Sample characteristics

\begin{tabular}{ll}
\hline Mean age (SD) & $12.56(2.86)$ \\
\hline 8-10.9 years (\%) & 36.2 \\
11-13.9 years (\%) & 24.1 \\
14-17.9 years (\%) & 39.7 \\
Sex (\% boys) & 55.2 \\
Race (\% yes) & \\
White & 41.4 \\
Black & 32.8 \\
Asian & 10.3 \\
American Indian & 1.7 \\
Other/mixed & 10.4 \\
Hispanic (\% yes) & 13.8 \\
Mean Framewise Displacement (SD) & $0.10(0.04)$ \\
Mean WASI FSIQ (SD) & $113.0(12.99)$ \\
Mean IUSC Total (SD) & $25.81(7.89)$ \\
Mean IUSC-12 Inhibitory (SD) & $9.03(3.49)$ \\
Mean IUSC-12 Prospective (SD) & $16.78(5.51)$ \\
Mean MASC Total Anxiety Score (SD) & $50.64(10.50)$ \\
\hline
\end{tabular}

Note: IUSC-12: Intolerance of Uncertainty Scale for Children, short version; WASI FSIQ: Weschler Abbreviated Scales of Intelligence Full Scale Intelligence Quotient; MASC: Multidimensional Anxiety Scale for Children.

Anxiety Scale for Children (MASC; March, Parker, Sullivan, Stallings, \& Conners, 1997). The MASC Total Anxiety score was used in the current study for follow up analyses examining the specificity of the association between IU and AI functional connectivity. Participants additionally completed the two subtest version of the Wechsler Abbreviated Scale of Intelligence (Matrix Reasoning and Vocabulary; Wechsler, 1999) to determine whether they met the minimum IQ of 75.

\section{Functional magnetic resonance imaging}

Data acquisition Scans were acquired using a 3.0T Siemens Allegra head-only MRI scanner (Siemens; Iselin, New Jersey) at the NYU Center for Brain Imaging. Each child completed a six- minute resting state scan of 35 contiguous $3 \mathrm{~mm}$ axial slices using a $\mathrm{T} 2 *$-sensitive gradient echo sequence $(\mathrm{TR}=$ $2000 \mathrm{~ms} ; \mathrm{TE}=30 ; \mathrm{FOV}=192 ; 64$ x 64 matrix; in-plane resolution $3 \times 3 \times 4$ ). Previous literature has found that sixminute resting scan is sufficient to offer a compromise between robust functional connectivity and experiment brevity (Van Dijk et al., 2009). A high-resolution T1-weighted MPRAGE image was acquired for each participant for anatomical registration.

Image preprocessing All brain pre-processing and group level analyses were conducted using an alpha version (0.3.9) of The 
Configurable Pipeline for the Analysis of Connectomes (CPAC; http://fcp-indi.github.io/). CPAC is a configurable, open-source, Nipype-based (http://nipy.org/nipype/), automated processing pipeline for resting state functional MRI data. Preprocessing consists of slice time correction (first slice as reference, interleaved acquisitions, Fourier interpolation), despiking (removal of extreme time series outliers), spatial smoothing (FWHM $=6 \mathrm{~mm})$, mean-based intensity normalization of all volumes by the same factor, temporal bandpass filtering to isolate low-frequency BOLD fluctuations of interest $(0.01-0.1 \mathrm{~Hz})$. Single-participant nuisance correction included 24 Friston motion parameters (Friston, Frith, Fletcher, Liddle, \& Frackowiak, 1996) and five CompCor signals (Behzadi, Restom, Liau, \& Liu, 2007). Structural and functional images were registered, coregistered, and normalized to a common stereotaxic space (Montreal Neurological Institute: MNI) using Boundary Based Registration (BBR) as implemented in FSL (Greve \& Fischl, 2009) and Advanced Normalization Tools (ANTs software; Avants et al., 2011); http://www.picsl.upenn.edu/ANTS/).

Seed-based correlation analyses Seed-based correlation analyses were conducted by using bilateral AI regions of interest (ROI) obtained from a previously published parcellation of the insula (Kelly et al., 2012). The mean time series of all voxels within the ROIs were computed by averaging the time series across all voxels within each ROI. Connectivity strength for each AI ROI was then assessed for each participant using a whole brain analysis in which correlations between each cluster's time series data was compared with all other voxels in the brain. This procedure produced individual participant level maps of all voxels demonstrating significant $\mathrm{iFC}$ with the $\mathrm{AI}$ (Gaussian random field [GRF] correction: $p<0.05, \mathrm{z}>2.3$ ). At the group level, three random-effects, ordinary least-squares models were used that included the IUSC-12 Total, IUSC-12 Prospective, or IUSC-12 Inhibitory as the variable of interest, and three nuisance covariates, age, sex, and mean frame-wise displacement, as computed per Jenkinson et al. (2002). Group analysis findings were GRF corrected at $p<0.05, \mathrm{z}>3.1$.

\section{Specificity analyses}

Although transdiagnostic in nature, IU often has been theorized to be related to anxiety disorders (Boswell, Thompson-Hollands, Farchione, \& Barlow, 2013; Carleton, 2016; Carleton et al., 2012; Chen, Yao, \& Qian, 2018; Correa, Liu, \& Shankman, 2019; Flores et al., 2018; McEvoy \& Erceg-Hurn, 2016; McEvoy \& Mahoney, 2012). Two approaches were taken to assess the specificity of the functional connectivity findings to IU. First, a more conservative method, given the small sample and moderate number of covariates, was to repeat the seed-based wholebrain analysis, including MASC Total Anxiety scores in the model. Given the expected correlation between IUSC12 and MASC Total Anxiety scores and limited power of this analysis, we also examined this in a post-hoc manner by testing the specificity of the associations between IU and $\mathrm{AI}$ iFC, using multiple linear regression analyses performed using SPSS 25.0 (IBM SPSS Statistics, Version 25.0 IBM Corp: Armonk, NY) for each significant cluster showing AI iFC differences. All models included age, sex, and mean FD as covariates. In the first model, IUSC-12 Inhibitory subscale Scores and MASC Total Anxiety scores were included as independent variables predicting left AI iFC. In the second model, IUSC-12 Inhibitory subscale scores and MASC Total Anxiety scores were included as independent variables predicting right $\mathrm{AI} \mathrm{iFC}$. Finally, IUSC-12 Prospective subscale scores and MASC Total Anxiety scores predicted left AI iFC. Because these analyses were conducted post hoc, IUSC-12 Prospective subscale score and anxiety predicting right $\mathrm{AI}$ connectivity was not probed, because no significant findings were observed in initial analyses.

\section{Results}

\section{Descriptive results}

Mean full-scale IQ scores were in the average range ( $M=$ 113.0; $S D=12.99)$. Mean scores on the IUSC-12 Total $(M$ $=25.81, S D=7.87)$, IUSC-12 Prospective $(M=16.78, S D=$ $5.51)$, and IUSC-12 Inhibitory $(M=9.03, S D=3.49)$ were consistent with those previously reported for nonanxious children and adolescents (Cornacchio et al., 2018). Mean MASC scores were in the expected range for a nonclinical sample $(M$ $=50.64 ; S D=10.50$ ). As expected, IUSC-12 variables correlated with each other and with MASC scores (Table 2). Mean framewise displacement (FD), a commonly used measure of head motion inside the scanner, correlated with IUSC-12 Inhibitory subscale score but did not correlate with any other variable of interest (Table 2).

\section{Whole-brain Al connectivity}

To confirm that AI connectivity was consistent with previous work and that the salience network could be detected, we examined the whole brain iFC for the left and right AI. As shown in the Supplementary Table 1 and Figure 1, findings were consistent with that observed in previous work (Kelly et al., 2012; Kurth, Zilles, Fox, Laird, \& Eickhoff, 2010).

\section{IU and Al connectivity analyses}

No significant associations were found between either left or right AI iFC and IUSC-12 Total scores. Significant 
Table 2. Correlation table for covariates and variables of interest

\begin{tabular}{|c|c|c|c|c|c|c|c|}
\hline & 1 & 2 & 3 & 4 & 5 & 6 & 7 \\
\hline 1. Age & - & & & & & & \\
\hline 2. Sex & -0.17 & - & & & & & \\
\hline 3. Mean FD & -0.21 & 0.02 & - & & & & \\
\hline 4. WASI FSIQ & -0.03 & 0.03 & -0.07 & - & & & \\
\hline 5. IUSC-12 Total Score & -0.19 & -0.02 & 0.22 & -0.25 & - & & \\
\hline 6. IUSC-12 Inhibitory subscale Score & -0.24 & -0.07 & $0.31 *$ & -0.26 & $0.80 * *$ & - & \\
\hline 7. IUSC-12 Prospective subscale Score & -0.12 & 0.02 & 0.11 & -0.19 & $0.93 * *$ & $0.51 * *$ & - \\
\hline 8. MASC Total Anxiety Score & -0.08 & 0.11 & 0.23 & -0.02 & $0.53 * *$ & $0.57 * *$ & $0.40 * *$ \\
\hline
\end{tabular}

Note: Mean FD: Mean Framewise Displacement; WASI FSIQ: Weschler Abbreviated Scales of Intelligence Full Scale Intelligence Quotient; IUSC-12: Intolerance of Uncertainty Scale for Children, short version; MASC: Multidimensional Anxiety Scale for Children.

$*<0.05 ; * *<0.01$.

associations were observed between the IUSC-12 Inhibitory subscale score and bilateral AI iFC. As shown in Fig. 1 and Table 3, IUSC-12 Inhibitory scores significantly predicted iFC between the left and right $\mathrm{AI}$ and right lateralized frontal regions, including the middle and inferior frontal gyri and precentral gyrus. A significant negative association was observed between IUSC-12 Prospective subscale scores and iFC between the left AI and midline cingulate regions including the paracingulate gyrus and ACC (Fig. 2; Table 3). No significant associations were found between the IUSC-12 Prospective subscale scores and right $\mathrm{AI}$ iFC. Further analysis regressing IUSC-12 Prospective subscale scores on connectivity between the right $\mathrm{AI}$ and the dACC region identified in the left AI analysis, controlling for age, sex, and mean FD, was performed. This analysis revealed a significant relationship ( $\beta$ $=-0.39, t=-3.73, p<0.001)$. Because this was not observed in the initial analysis using stringent Gaussian random field correction, it is likely that this relationship is weaker than that observed for the left AI.

\section{Specificity analyses}

In an initial effort to examine the specificity of the association between IUSC-12 subscale scores and AI iFC, seed-based analyses were repeated with MASC Total Anxiety scores in the model. This analysis only included 55 participants who had MASC scores available. In these analyses, no significant associations were found between IUSC-12 Inhibitory subscale scores or IUSC-12 Prospective subscale scores and the left or right $\mathrm{AI}$ iFC.

There is no clear way to determine whether the loss of significant AI iFC findings related to IUSC-12 scores suggests a lack of specificity of these results or a lack of power by inclusion of an additional covariate that is moderately correlated with IUSC-12 scores. We conducted less stringent posthoc analyses using multiple linear regression analyses, including MASC Total Anxiety scores for each of the significant AI iFC clusters identified in the first whole-brain seed-based correlation analysis. As seen in Table 4, IUSC-12 Inhibitory subscale scores remained significant predictors of both left $(\beta=$

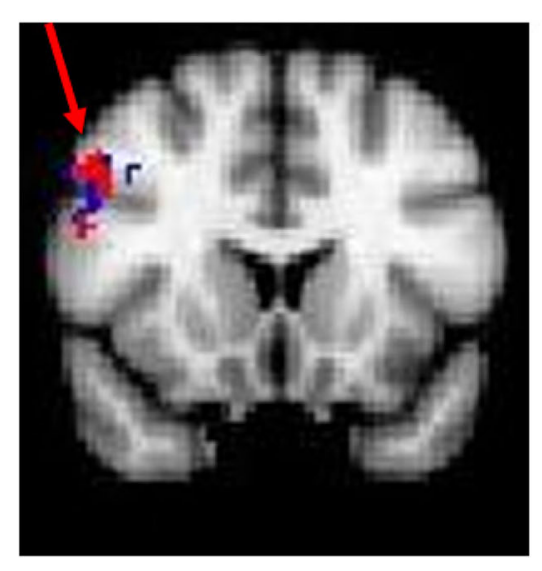

Fig. 1. Anterior insula connectivity associated with inhibitory intolerance of uncertainty. Clusters of significant $\mathrm{iFC}$ with left and right anterior insula predicted by IUSC-12 Inhibitory scores. Blue indicates iFC with

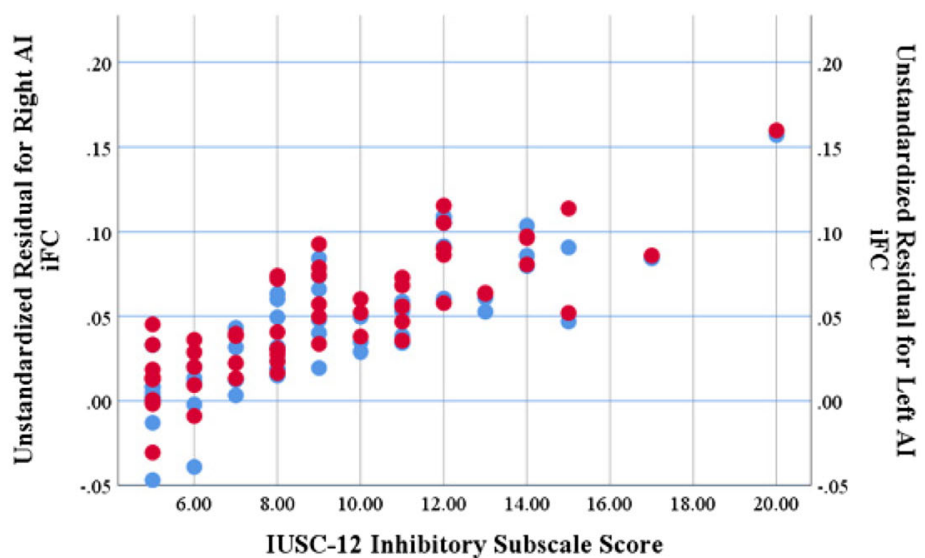

right anterior insula; Red indicates $\mathrm{iFC}$ with left anterior insula. Note: iFC: intrinsic functional connectivity; AI: Anterior Insula; IUSC-12: Intolerance of Uncertainty Scale- Child Version, short version 
Table 3. Locations of anterior insula $\mathrm{iFC}$ significantly predicted by intolerance of uncertainty

\begin{tabular}{lllll}
\hline & \# voxels & $\mathrm{x}$ & $\mathrm{y}$ & $\mathrm{z}$ \\
\hline Inhibitory subscale & & & & \\
$\quad$ Left AI - Right MFG/IFG & 100 & 54 & 16 & 38 \\
$\quad$ Right AI - Right MFG/IFG & 79 & 54 & 20 & 26 \\
$\begin{array}{l}\text { Prospective subscale } \\
\quad \text { Left AI - dACC }\end{array}$ & 150 & 6 & 26 & 32 \\
\hline
\end{tabular}

Note: All coordinates are normalized to MNI space. Voxel size $=3 \times 3 \times$ 4. AI: Anterior Insula; MFG/IGF: Medial Frontal Gyrus/Inferior Frontal Gyrus; dACC: dorsal Anterior Cingulate Cortex.

0.46, $t=2.99, p=0.004)$ and right $(\beta=0.51, t=3.5, p=$ 0.001 ) AI findings after controlling for MASC Total Anxiety score. MASC Total Anxiety scores did not significantly contribute to the model. Similar results were found for the third model including IUSC-12 Prospective subscale and MASC Total Anxiety score predicting left AI iFC. IUSC-12 Prospective subscale scores predicted left AI-ACC iFC ( $\beta=$ $-0.55, t=-4.69, p<0.000)$ after controlling for MASC Total Anxiety scores, whereas MASC score did not significantly contribute to the model.

\section{Discussion}

The present investigation assessed the functional connectivity of the anterior insula as it relates to intolerance of uncertainty in healthy children and adolescents. Whereas the IUSC-12 Total Score (which pools together items on Inhibitory and Prospective IU) was not found to be associated with AI iFC, significant associations were observed for the two subscales. Youth with higher Inhibitory IU demonstrated increased iFC between the anterior insula and right frontal regions. Higher Prospective IU was associated with decreased functional connectivity between the anterior insula and the anterior cingulate. Although these findings were no longer evident after including anxiety in the whole-brain models, less conservative post-hoc specificity analyses suggest that IU may uniquely contribute to $\mathrm{AI}$ iFC with specific regions, over and above anxiety. Thus, while additional work with larger samples is clearly needed, these findings contribute to a growing body of research suggesting that domains of IU are associated with alterations in AI function (Gorka et al., 2016; Shankman et al., 2014).

Inhibitory IU was predictive of iFC between the bilateral AI and regions of the right inferior/middle frontal gyri, which are typically considered part of the central executive or frontoparietal network (Uddin, 2015; Yeo et al., 2011). A recent task-based meta-analysis suggests that coactivation between the $\mathrm{AI}$ and these regions of the frontal cortex, particularly in the right hemisphere, is important for self-regulation, including both behavioral and emotional control (Langner, Leiberg, Hoffstaedter, \& Eickhoff, 2018). In particular, within this self-regulation network, coactivation between the right lateralized inferior and middle frontal gyri and the AI is associated with top-down attentional control, tracking event irregularities, and detecting emotionally salient distractors (Blair et al., 2007; Luo et al., 2007; Vossel, Weidner, \& Fink, 2011). Furthermore, activation in the right interior and middle frontal gyri has been implicated in responding specifically to event irregularities that require an inhibitory response (Chikazoe et al., 2008). Thus, the greater $\mathrm{iFC}$ observed in youth with higher Inhibitory IU scores may reflect their experience of uncertainty as particularly emotionally salient. In such situations, individuals with high Inhibitory IU may be less able to redirect their attention to access alternative coping strategies resulting in "uncertainty paralysis." Furthermore, executive control mediated by these regions of prefrontal cortex is important for many daily tasks, including decision making, which often is impaired in individuals high in IU (Carleton et al., 2016; Doruk, Dugenci, Ersoz, \& Oznur, 2015). In fact, clinical theorists suggest that impairment in decision making associated with IU may underlie the development
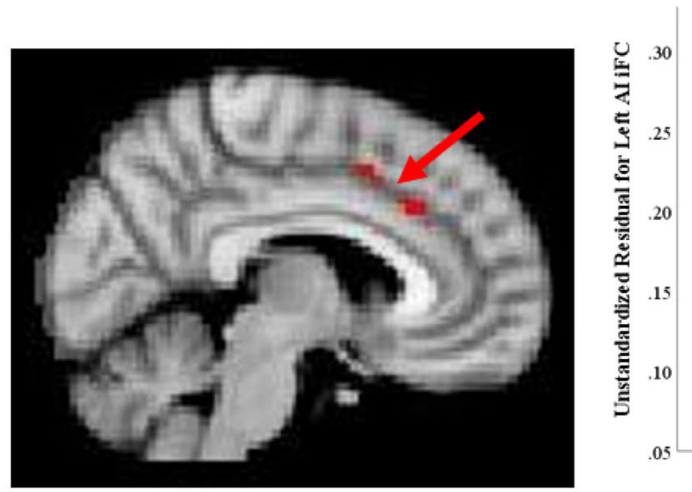

Fig. 2. Anterior Insula Connectivity Associated with Prospective Intolerance of Uncertainty. Clusters of significant $\mathrm{iFC}$ with left anterior insula predicted by IUSC-12 Prospective scores. Note: iFC: intrinsic functional connectivity; AI: Anterior Insula; IUSC-12: Intolerance of Uncertainty Scale- Child Version, short version 
Table 4. Results from specificity analyses

\begin{tabular}{llr}
\hline Regression Model* & IUSC-12 Subscale $\beta(p)$ & MASC Total Anxiety $\beta(p)$ \\
\hline Inhibitory subscale & & 0.13 (NS) \\
$\quad$ Left AI - MFG/IFG iFC & $0.46(0.004)$ & 0.05 (NS) \\
$\quad$ Right AI - MFG/IFG iFC & $0.51(0.001)$ & -0.11 (NS) \\
Prospective subscale & & $-0.55(<0.00)$ \\
$\quad$ Left AI - ACC iFC & & \\
\hline
\end{tabular}

*All models include covariates age, sex, and mean framewise displacement.

Note: IUSC-12: Intolerance of Uncertainty Scale for Children, short version; AI: Anterior Insula; MFG/IGF: Medial Frontal Gyrus/Inferior Frontal Gyrus; dACC: dorsal Anterior Cingulate Cortex.

of anxiety disorders from high trait IU (Grupe \& Nitschke, 2013). Further investigations are needed to test whether alterations in the $\mathrm{iFC}$ between $\mathrm{AI}$ and the right inferior/middle frontal gyri impact decision making in support of this model.

Prospective IU was predictive of decreased iFC between the AI and the AACC, supporting study hypotheses that iFC of these regions would be associated with IU. Although our findings are inconsistent with some adult work that has found increases in AI-dACC connectivity associated with decision making avoidance (Jung et al., 2014), these results are consistent with previous studies that find reduced AI-dACC iFC associated with anxiety in clinical (Xu et al., 2018) and nonclinical (Geng, Li, Chen, Li, \& Gu, 2015) samples of adolescents. Furthermore, task-based work has shown increased activation in the AACC associated with both IU and uncertainty during a decision-making task in adolescents that was not observed in adults, suggesting that neural representations of IU may differ across development (Krain et al., 2008; Krain et al., 2006). The AI and the dACC belong to the salience network, a group of regions important for decision making, ambiguity, and novelty (Uddin, 2017). Decreases in connectivity between the AI and dACC associated with increased IU may underlie deficits in the ability to regulate attention away from future-oriented, abstract threat. In light of findings that Prospective IU is consistently associated with increased worry and the use of worry as a maladaptive coping mechanism (Dugas, Gosselin, \& Ladouceur, 2001; Flores et al., 2018), this decreased coupling of AI and AACC may contribute to the development of maladaptive worry in youth.

Conservative specificity analyses, including self-reported anxiety, in the models of IU predicting anterior insula connectivity resulted in a loss of significant findings. This could imply that the findings observed in the current study reflect broader anxiety-related connectivity patterns rather than the contribution of IU specifically. Less conservative, post-hoc specificity analyses found no effect of anxiety, suggesting that IU may uniquely predict anterior insula connectivity with specific regions. This latter finding is in line with behavioral studies that suggest that IU and anxiety are unique constructs (Dugas \& Koerner, 2005; Dugas \& Ladouceur, 2000). Further studies with larger samples are needed to tease out the unique associations of IU and anxiety with anterior insula network connectivity.

This study did not observe differences in $\mathrm{AI}$ iFC associated with the IUSC-12 Total Score. Although two studies of Dutch teens (Boelen et al., 2010; Dekkers et al., 2017) have supported the utility of a general IU factor, one study evaluating IU factor structure across a broad age range (9-18 years) found that a single factor structure of IU in children and adolescents showed poor fit among parent-reported data and "mixed" to "good" fit among child-reported data (Cornacchio et al., 2018). This supports the notion that youth IU may be better represented by the two subfactors of IU, rather than by a general factor and may explain the null results observed in this study. These findings also highlight the need for more work in this area to better understand IU across the developmental spectrum.

This study is not without its limitations. The small sample size of this preliminary evaluation precluded our ability to perform a more comprehensive examination of how age, sex, pubertal development status, and anxiety may differentially contribute to the relationship between IU and AI iFC. Additionally, due to the lack of previous research, power analyses could not be adequately performed, and this study may have been underpowered to detect more nuanced relationships between IU and AI connectivity, particularly when controlling for anxiety. Relatively modest internal consistency in Inhibitory IU subscale scores, perhaps due to fewer number of items in this subscale (Streiner, Norman, \& Cairney, 2015), also may have affected findings. There has been in shift in the field toward longer resting state scans. It is possible that the short scan length may have biased the data (O'Connor et al., 2017). Finally, the data presented represent associations observed in small sample of healthy children and adolescents; investigating IU among larger, clinical populations could substantiate the connection between IU and the development and maintenance of psychopathology. Additionally, future studies should include behavioral measures of IU, both in and out of the scanner, to support child self-report and to assess functional connectivity during moments of uncertainty that might be associated with elevations in IU. 
The current study suggests that youth with high IU demonstrate stronger positive iFC between the AI and frontal regions, and negative iFC within the salience network, and that these associations vary across key domains of IU. It is possible that such alterations in salience network functioning underlie the vulnerability connecting IU to the development and maintenance of psychopathology. Thus, these findings have important, although preliminary, clinical implications. If alterations in salience network functioning underlie vulnerability for IU, then it might follow that salience networking functioning could serve as one early neural marker for risk for the development of psychopathology. Longitudinal studies of youth with and without clinical diagnoses are needed to validate these implications. IU is an important transdiagnostic marker of risk for, and maintenance of, psychopathology among healthy youth (Boelen et al., 2010; Dugas et al., 2012; Laugesen, Dugas, \& Bukowski, 2003; Osmanagaoglu, Creswell, \& Dodd, 2018). The present findings suggest that alterations in AI functional connectivity associated with elevated Inhibitory and Prospective IU support further investigation of IU as a potential neurophysiological marker of risk for the development of psychopathology in youth.

Acknowledgements Research support provided by the National Institute of Mental Health (K23MH087770 to A.D.M; R01MH081218 to F. Xavier Castellanos; K23MH074821 to A.K. Roy), from the National Institute of Child Health and Human Development (R01HD065282 to F.X.C.), the Stavros Niarchos Foundation (awarded to F.X.C.), and the Brain and Behavior Research Foundation (NARSAD Young Investigator Award to A.K. Roy).

Open practices statement None of the data or materials for the experiments reported here is publicly available, and none of the experiments was preregistered.

\section{Compliance with ethical standards}

Disclosures The authors report no financial disclosures or conflicts of interest.

\section{References}

Abrams, D. A., Padmanabhan, A., Chen, T., Odriozola, P., Baker, A. E., Kochalka, J., ... Menon, V. (2019). Impaired voice processing in reward and salience circuits predicts social communication in children with autism. Elife, 8, e39906. https://doi.org/10.7554/eLife. 39906

Avants, B. B., Tustison, N. J., Song, G., Cook, P. A., Klein, A., \& Gee, J. C. (2011). A reproducible evaluation of ANTs similarity metric performance in brain image registration. Neuroimage, 54, 2033-2044. https://doi.org/10.1016/j.neuroimage.2010.09.025

Banducci, A. N., Bujarski, S. J., Bonn-Miller, M. O., Patel, A., \& Connolly, K. M. (2016). The impact of intolerance of emotional distress and uncertainty on veterans with co-occurring PTSD and substance use disorders. Journal of Anxiety Disorders, 41, 73-81. https://doi.org/10.1016/j.janxdis.2016.03.003
Bar-Anan, Y., Wilson, T. D., \& Gilbert, D. T. (2009). The feeling of uncertainty intensifies affective reactions. Emotion, 9, 123-127. https://doi.org/10.1037/a0014607

Barahmand, U. (2008). Age and gender differences in adolescent worry. Personality and Individual Differences, 45, 778-783. https://doi.org/ 10.1016/j.paid.2008.08.006

Behzadi, Y., Restom, K., Liau, J., \& Liu, T. T. (2007). A component based noise correction method (CompCor) for BOLD and perfusion based fMRI. Neuroimage, 37, 90-101. https://doi.org/10.1016/j. neuroimage.2007.04.042

Blair, K. S., Smith, B. W., Mitchell, D. G., Morton, J., Vythilingam, M., Pessoa, L., ... Blair, R. J. (2007). Modulation of emotion by cognition and cognition by emotion. Neuroimage, 35, 430-440. https:// doi.org/10.1016/j.neuroimage.2006.11.048

Boelen, P. A., Vrinssen, I., \& van Tulder, F. (2010). Intolerance of uncertainty in adolescents: correlations with worry, social anxiety, and depression. Journal of Nervous and Mental Disease, 198, 194200. https://doi.org/10.1097/NMD.0b013e3181d143de

Boswell, J. F., Thompson-Hollands, J., Farchione, T. J., \& Barlow, D. H. (2013). Intolerance of uncertainty: a common factor in the treatment of emotional disorders. Journal of Clinical Psychology, 69, 630645. https://doi.org/10.1002/jclp.21965

Carleton, R. N. (2016). Into the unknown: A review and synthesis of contemporary models involving uncertainty. Journal of Anxiety Disorders, 39, 30-43. https://doi.org/10.1016/j.janxdis.2016.02.007

Carleton, R. N., Duranceau, S., Shulman, E. P., Zerff, M., Gonzales, J., \& Mishra, S. (2016). Self-reported intolerance of uncertainty and behavioural decisions. Journal of Behavior Therapy and Experimental Psychiatry, 51, 58-65. https://doi.org/10.1016/j.jbtep.2015.12.004

Carleton, R. N., Norton, M. A., \& Asmundson, G. J. (2007). Fearing the unknown: a short version of the Intolerance of Uncertainty Scale. Journal of Anxiety Disorders, 21, 105-117. https://doi.org/10.1016/j. janxdis.2006.03.014

Carleton, R. N., Mulvogue, M. K., Thibodeau, M. A., McCabe, R. E., Antony, M. M., \& Asmundson, G. J. (2012). Increasingly certain about uncertainty: Intolerance of uncertainty across anxiety and depression. Journal of Anxiety Disorders, 26, 468-479. https://doi.org/ 10.1016/j.janxdis.2012.01.011

Chen, S., Yao, N., \& Qian, M. (2018). The influence of uncertainty and intolerance of uncertainty on anxiety. Journal of Behavior Therapy and Experimental Psychiatry, 61, 60-65. https://doi.org/10.1016/j. jbtep.2018.06.005

Chikazoe, J., Jimura, K., Asari, T., Yamashita, K.-I., Morimoto, H., Hirose, S., ... Konishi, S. (2008). Functional Dissociation in Right Inferior Frontal Cortex during Performance of Go/No-Go Task. Cerebral Cortex, 19, 146-152. https://doi.org/10.1093/cercor/ bhn065

Comer, J. S., Roy, A. K., Furr, J. M., Gotimer, K., Beidas, R. S., Dugas, M. J., \& Kendall, P. C. (2009). The intolerance of uncertainty scale for children: a psychometric evaluation. Psychol Assess, 21, 402411. https://doi.org/10.1037/a0016719

Cornacchio, D., Sanchez, A. L., Coxe, S., Roy, A., Pincus, D. B., Read, K. L., ... Comer, J. S. (2018). Factor structure of the intolerance of uncertainty scale for children. Journal of Anxiety Disorders, 53, 100-107. https://doi.org/10.1016/j.janxdis.2017.07.003

Correa, K. A., Liu, H., \& Shankman, S. A. (2019). The role of intolerance of uncertainty in current and remitted internalizing and externalizing psychopathology. Journal of Anxiety Disorders, 62, 68-76. https:// doi.org/10.1016/j.janxdis.2019.01.001

Dekkers, L. M. S., Jansen, B. R. J., Salemink, E., \& Huizenga, H. M. (2017). Intolerance of Uncertainty Scale: Measurement invariance among adolescent boys and girls and relationships with anxiety and risk taking. Journal of Behavior Therapy and Experimental Psychiatry, 55, 57-65. https://doi.org/10.1016/j.jbtep.2016.11.009

Doruk, A., Dugenci, M., Ersoz, F., \& Oznur, T. (2015). Intolerance of Uncertainty and Coping Mechanisms in Nonclinical Young 
Subjects. Noropsikiyatri Arsivi, 52, 400-405. https://doi.org/10. 5152/npa.2015.8779

Dugas, M. J., \& Koerner, N. (2005). Cognitive-Behavioral Treatment for Generalized Anxiety Disorder: Current Status and Future Directions. Journal of Cognitive Psychotherapy, 19, 61-81. https:// doi.org/10.1891/jcop.19.1.61.66326

Dugas, M. J., Gosselin, P., \& Ladouceur, R. (2001). Intolerance of Uncertainty and Worry: Investigating Specificity in a Nonclinical Sample. Cognitive Therapy and Research, 25, 551-558. https://doi. org/10.1023/a:1005553414688

Dugas, M. J., Laugesen, N., \& Bukowski, W. M. (2012). Intolerance of uncertainty, fear of anxiety, and adolescent worry. Journal of Abnormal Child Psychology, 40, 863-870. https://doi.org/10.1007/ s10802-012-9611-1

Dugas, M. J., \& Ladouceur, R. (2000). Treatment of GAD. Targeting intolerance of uncertainty in two types of worry. Behavior Modification, 24, 635-657. https://doi.org/10.1177/ 0145445500245002

Eckert, M. A., Menon, V., Walczak, A., Ahlstrom, J., Denslow, S., Horwitz, A., \& Dubno, J. R. (2009). At the heart of the ventral attention system: the right anterior insula. Human Brain Mapping, 30, 2530-2541. https://doi.org/10.1002/hbm.20688

Einstein, D. A. (2014). Extension of the Transdiagnostic Model to Focus on Intolerance of Uncertainty: A Review of the Literature and Implications for Treatment. Clinical Psychology, 21, 280-300. https://doi.org/10.1111/cpsp.12077

Flores, A., Lopez, F. J., Vervliet, B., \& Cobos, P. L. (2018). Intolerance of uncertainty as a vulnerability factor for excessive and inflexible avoidance behavior. Behaviour Research and Therapy, 104, 34-43. https://doi.org/10.1016/j.brat.2018.02.008

Freeston, M. H., Rhéaume, J., Letarte, H., Dugas, M. J., \& Ladouceur, R. (1994). Why do people worry? Personality and Individual Differences, 17, 791-802. https://doi.org/10.1016/0191-8869(94) 90048-5

Friston, K. J., Frith, C. D., Fletcher, P., Liddle, P. F., \& Frackowiak, R. S. (1996). Functional topography: multidimensional scaling and functional connectivity in the brain. Cerebral Cortex, 6, 156-164.

Geng, H., Li, X., Chen, J., Li, X., \& Gu, R. (2015). Decreased Intra- and Inter-Salience Network Functional Connectivity is Related to Trait Anxiety in Adolescents. Frontiers in Behavioral Neuroscience, 9, 110. https://doi.org/10.3389/fnbeh.2015.00350

Goodkind, M., Eickhoff, S. B., Oathes, D. J., Jiang, Y., Chang, A., JonesHagata, L. B., ... Etkin, A. (2015). Identification of a common neurobiological substrate for mental illness. JAMA Psychiatry, 72, 305-315. https://doi.org/10.1001/jamapsychiatry.2014.2206

Gorka, S. M., Nelson, B. D., Phan, K. L., \& Shankman, S. A. (2016). Intolerance of uncertainty and insula activation during uncertain reward. Cognitive, Affective \& Behavioral Neuroscience, 16, 929939. https://doi.org/10.3758/s13415-016-0443-2

Gramszlo, C., Fogleman, N. D., Rosen, P. J., \& Woodruff-Borden, J. (2018). Intolerance of uncertainty in children with attention-deficit/ hyperactivity disorder. Atten Defic Hyperact Disord, 10, 189-197. https://doi.org/10.1007/s12402-017-0244-7

Greve, D. N., \& Fischl, B. (2009). Accurate and robust brain image alignment using boundary-based registration. Neuroimage, 48, 6372. https://doi.org/10.1016/j.neuroimage.2009.06.060

Grist, R. M., \& Field, A. P. (2012). The mediating effect of cognitive development on children's worry elaboration. Journal of Behavior Therapy and Experimental Psychiatry, 43, 801-807. https://doi.org/ 10.1016/j.jbtep.2011.11.002

Grupe, D. W., \& Nitschke, J. B. (2013). Uncertainty and anticipation in anxiety: an integrated neurobiological and psychological perspective. Nature Reviews: Neuroscience, 14, 488-501. https://doi.org/10. 1038/nrn3524

Han, W., Sorg, C., Zheng, C., Yang, Q., Zhang, X., Ternblom, A., ... Shao, J. (2019). Low-rank network signatures in the triple network separate schizophrenia and major depressive disorder. NeuroImage: Clinical, 22, 101725 101721-101711. https://doi.org/10.1016/j.nicl. 2019.101725

Hunt, C., Cooper, S. E., Hartnell, M. P., \& Lissek, S. (2019). Anxiety sensitivity and intolerance of uncertainty facilitate associations between generalized Pavlovian fear and maladaptive avoidance decisions. Journal of Abnormal Psychology. https://doi.org/10.1037/ abn0000422

Jenkinson, M., Bannister, P., Brady, M., \& Smith, S. (2002). Improved optimization for the robust and accurate linear registration and motion correction of brain images. Neuroimage, 17, 825-841. https:// doi.org/10.1006/nimg.2002.1132

Jung, Y. C., Schulte, T., Muller-Oehring, E. M., Hawkes, W., Namkoong, K., Pfefferbaum, A., \& Sullivan, E. V. (2014). Synchrony of anterior cingulate cortex and insular-striatal activation predicts ambiguity aversion in individuals with low impulsivity. Cerebral Cortex, 24, 1397-1408. https://doi.org/10.1093/cercor/bht008

Kaufman, J., Birmaher, B., Brent, D., Rao, U., Flynn, C., Moreci, P., ... Ryan, N. (1997). Schedule for Affective Disorders and Schizophrenia for School-Age Children-Present and Lifetime Version (K-SADS-PL): initial reliability and validity data. Journal of the American Academy of Child and Adolescent Psychiatry, 36, 980-988. https://doi.org/10.1097/00004583-199707000-00021

Kelly, C., Toro, R., Di Martino, A., Cox, C. L., Bellec, P., Castellanos, F. X., \& Milham, M. P. (2012). A convergent functional architecture of the insula emerges across imaging modalities. Neuroimage, 61, 1129-1142. https://doi.org/10.1016/j.neuroimage.2012.03.021

Kesby, A., Maguire, S., Brownlow, R., \& Grisham, J. R. (2017). Intolerance of Uncertainty in eating disorders: An update on the field. Clinical Psychology Review, 56, 94-105. https://doi.org/10. 1016/j.cpr.2017.07.002

Kessler, R. C., Berglund, P., Demler, O., Jin, R., Merikangas, K. R., \& Walters, E. E. (2005). Lifetime prevalence and age-of-onset distributions of DSM-IV disorders in the National Comorbidity Survey Replication. Archives of General Psychiatry, 62, 593-602. doi: https://doi.org/10.1001/archpsyc.62.6.593

Krain, A. L., Gotimer, K., Hefton, S., Ernst, M., Castellanos, F. X., Pine, D. S., \& Milham, M. P. (2008). A functional magnetic resonance imaging investigation of uncertainty in adolescents with anxiety disorders. Biological Psychiatry, 63, 563-568. https://doi.org/10. 1016/j.biopsych.2007.06.011

Krain, A. L., Hefton, S., Pine, D. S., Ernst, M., Castellanos, F. X., Klein, R. G., \& Milham, M. P. (2006). An fMRI examination of developmental differences in the neural correlates of uncertainty and decision-making. Journal of Child Psychology and Psychiatry and Allied Disciplines, 47, 1023-1030. https://doi.org/10.1111/j.14697610.2006.01677.x

Kurth, F., Zilles, K., Fox, P. T., Laird, A. R., \& Eickhoff, S. B. (2010). A link between the systems: functional differentiation and integration within the human insula revealed by meta-analysis. Brain Structure \& Function, 214, 519-534. https://doi.org/10.1007/s00429-010-0255-z

Langner, R., Leiberg, S., Hoffstaedter, F., \& Eickhoff, S. B. (2018). Towards a human self-regulation system: Common and distinct neural signatures of emotional and behavioural control. Neuroscience and Biobehavioral Reviews, 90, 400-410. https://doi.org/10.1016/j. neubiorev.2018.04.022

Laugesen, N., Dugas, M. J., \& Bukowski, W. M. (2003). Understanding adolescent worry: the application of a cognitive model. Journal of Abnormal Child Psychology, 31, 55-64. http:// dx.doi.org.avoserv2.library.fordham.edu/10.1023/A:1021721332181

Luhmann, C. C., Ishida, K., \& Hajcak, G. (2011). Intolerance of uncertainty and decisions about delayed, probabilistic rewards. Behavior Therapy, 42, 378-386. https://doi.org/10.1016/j.beth.2010.09.002

Luo, Q., Mitchell, D., Jones, M., Mondillo, K., Vythilingam, M., \& Blair, R. J. (2007). Common regions of dorsal anterior cingulate and prefrontal-parietal cortices provide attentional control of distracters 
varying in emotionality and visibility. Neuroimage, 38, 631-639. https://doi.org/10.1016/j.neuroimage.2007.07.051

Mahoney, A. E., \& McEvoy, P. M. (2012). A transdiagnostic examination of intolerance of uncertainty across anxiety and depressive disorders. Cognitive Behaviour Therapy, 41, 212-222. https://doi.org/ 10.1080/16506073.2011.622130

Manoliu, A., Meng, C., Brandl, F., Doll, A., Tahmasian, M., Scherr, M., ... Sorg, C. (2013). Insular dysfunction within the salience network is associated with severity of symptoms and aberrant inter-network connectivity in major depressive disorder. Frontiers in Human Neuroscience, 7, 1-17. https://doi.org/10.3389/fnhum.2013.00930

March, J. S., Parker, J. D., Sullivan, K., Stallings, P., \& Conners, C. K. (1997). The Multidimensional Anxiety Scale for Children (MASC): factor structure, reliability, and validity. Journal of the American Academy of Child and Adolescent Psychiatry, 36, 554-565. https:// doi.org/10.1097/00004583-199704000-00019

McEvoy, P. M., \& Erceg-Hurn, D. M. (2016). The search for universal transdiagnostic and trans-therapy change processes: Evidence for intolerance of uncertainty. Journal of Anxiety Disorders, 41, 96107. https://doi.org/10.1016/j.janxdis.2016.02.002

McEvoy, P. M., \& Mahoney, A. E. (2012). To be sure, to be sure: intolerance of uncertainty mediates symptoms of various anxiety disorders and depression. Behavior Therapy, 43, 533-545. https://doi.org/ 10.1016/j.beth.2011.02.007

Menon, V. (2011). Large-scale brain networks and psychopathology: a unifying triple network model. Trends in Cognitive Sciences, 15, 483-506. https://doi.org/10.1016/j.tics.2011.08.003

Merikangas, K. R., He, J. P., Burstein, M., Swendsen, J., Avenevoli, S., Case, B., ... Olfson, M. (2011). Service utilization for lifetime mental disorders in U.S. adolescents: results of the National Comorbidity Survey-Adolescent Supplement (NCS-A). Journal of the American Academy of Child and Adolescent Psychiatry, 50, 32-45. https://doi. org/10.1016/j.jaac.2010.10.006

O'Connor, D., Potler, N. V., Kovacs, M., Xu, T., Ai, L., Pellman, J., ... Milham, M. P. (2017). The Healthy Brain Network Serial Scanning Initiative: a resource for evaluating inter-individual differences and their reliabilities across scan conditions and sessions. GigaScience, 6, 1-14. https://doi.org/10.1093/gigascience/giw011

Osmanagaoglu, N., Creswell, C., \& Dodd, H. F. (2018). Intolerance of Uncertainty, anxiety, and worry in children and adolescents: A metaanalysis. Journal of Affective Disorders, 225, 80-90. https://doi.org/ 10.1016/j.jad.2017.07.035

Peters, S. K., Dunlop, K., \& Downar, J. (2016). Cortico-Striatal-Thalamic Loop Circuits of the Salience Network: A Central Pathway in Psychiatric Disease and Treatment. Frontiers in Systems Neuroscience, 10, 104. https://doi.org/10.3389/fnsys.2016.00104

Phan, K. L., Wager, T., Taylor, S. F., \& Liberzon, I. (2002). Functional neuroanatomy of emotion: a meta-analysis of emotion activation studies in PET and fMRI. Neuroimage, 16, 331-348. https://doi. org/10.1006/nimg.2002.1087

Schienle, A., Kochel, A., Ebner, F., Reishofer, G., \& Schafer, A. (2010). Neural correlates of intolerance of uncertainty. Neuroscience Letters, 479, 272-276. https://doi.org/10.1016/j.neulet.2010.05.078

Seeley, W. W., Menon, V., Schatzberg, A. F., Keller, J., Glover, G. H., Kenna, H., ... Greicius, M. D. (2007). Dissociable intrinsic connectivity networks for salience processing and executive control. Journal of Neuroscience, 27, 2349-2356. https://doi.org/10.1523/ JNEUROSCI.5587-06.2007

Shankman, S. A., Gorka, S. M., Nelson, B. D., Fitzgerald, D. A., Phan, K. L., \& O'Daly, O. (2014). Anterior insula responds to temporally unpredictable aversiveness: an fMRI study. Neuroreport, 25, 596600. https://doi.org/10.1097/WNR.0000000000000144

Silverman, W. K., \& Albano, A. M. (1996). The Anxiety Disorders Interview Schedule for Children for DSM-IV, Child and Parent Versions. San Antonio, TX: Psychological Corporation.
Simmons, A., Matthews, S. C., Paulus, M. P., \& Stein, M. B. (2008). Intolerance of uncertainty correlates with insula activation during affective ambiguity. Neuroscience Letters, 430, 92-97. https://doi. org/10.1016/j.neulet.2007.10.030

Steinberg, L., \& Morris, A. S. (2001). Adolescent development. Annual Review of Psychology, 52, 83-110. https://doi.org/10.1146/annurev. psych.52.1.83

Streiner, D. L., Norman, G. R., \& Cairney, J. (2015). Health measurement scales: A practical guide to their development and use, 5 th ed. New York, NY: Oxford University Press. https://doi.org/10.1111/17536405.12484

Tolin, D. F., Abramowitz, J. S., Brigidi, B. D., \& Foa, E. B. (2003). Intolerance of uncertainty in obsessive-compulsive disorder. Journal of Anxiety Disorders, 17, 233-242. https://doi.org/10.1016/ S0887-6185(02)00182-2

Uddin, L. Q. (2017). Salience network of the human brain. San Diego, California: Academic Press. https://doi.org/10.1016/C2015-0-01862-7

Uddin, L. Q. (2015). Salience processing and insular cortical function and dysfunction. Nature Reviews Neuroscience, 55. https://doi.org/10. 1038/nrn3857

Van Dijk, K. R. A., Hedden, T., Venkataraman, A., Evans, K. C., Lazar, S. W., \& Buckner, R. L. (2009). Intrinsic Functional Connectivity As a Tool For Human Connectomics: Theory, Properties, and Optimization. Journal of Neurophysiology, 103, 297-321. https:// doi.org/10.1152/jn.00783.2009

Vasey, M. W., \& Crnic, K. A. (1994). Worry in childhood: A developmental perspective. Cognitive Therapy and Research, 18, 529-549. https://doi.org/10.1007/BF02355667

Vossel, S., Weidner, R., \& Fink, G. R. (2011). Dynamic coding of events within the inferior frontal gyrus in a probabilistic selective attention task. Journal of Cognitive Neuroscience 23, 414-424. https://doi. org/10.1162/jocn.2010.21441

Wechsler, D. (1999). Wechsler Abbreviated Scale of Intelligence (WASI). San Antonio, TX: Psychological Corporation.

Wever, M., Smeets, P., \& Sternheim, L. (2015). Neural Correlates of Intolerance of Uncertainty in Clinical Disorders. Journal of Neuropsychiatry and Clinical Neurosciences, 27, 345-353. https:// doi.org/10.1176/appi.neuropsych.14120387

White, R. G., \& Gumley, A. (2010). Intolerance of uncertainty and distress associated with the experience of psychosis. Psychology and Psychotherapy: Theory, Research and Practice, 83, 317-324. https://doi.org/10.1348/147608309X477572

White, T. P., Engen, N. H., Sørensen, S., Overgaard, M., \& Shergill, S. S. (2014). Uncertainty and confidence from the triple-network perspective: Voxel-based meta-analyses. Brain and Cognition, 85, 191-200. https://doi.org/10.1016/j.bandc.2013.12.002

Xu, J., Van Dam, N. T., Feng, C., Luo, Y., Ai, H., Gu, R., \& Xu, P. (2018). Anxious brain networks: A coordinate-based activation likelihood estimation meta-analysis of resting-state functional connectivity studies in anxiety. Neuroscience and Biobehavioral Reviews, 96, 21-30. https://doi.org/10.1016/j.neubiorev.2018.11.005

Yang, Y., Liu, S., Jiang, X., Yu, H., Ding, S., Lu, Y., ... Lv, L. (2019). Common and Specific Functional Activity Features in Schizophrenia, Major Depressive Disorder, and Bipolar Disorder. Frontiers in Psychiatry, 10, 52. https://doi.org/10.3389/fpsyt.2019.00052

Yeo, B. T., Krienen, F. M., Sepulcre, J., Sabuncu, M. R., Lashkari, D., Hollinshead, M., ... Buckner, R. L. (2011). The organization of the human cerebral cortex estimated by intrinsic functional connectivity. Journal of Neurophysiology, 106, 1125-1165. https://doi.org/10. 1152/jn.00338.2011

Publisher's note Springer Nature remains neutral with regard to jurisdictional claims in published maps and institutional affiliations. 